Tropical Journal of Pharmaceutical Research February 2016; 15 (2): 267-274

ISSN: $1596-5996$ (print); 1596-9827 (electronic) (c) Pharmacotherapy Group, Faculty of Pharmacy, University of Benin, Benin City, 300001 Nigeria.

\title{
A Study on Expression and Tyrosine 705 phosphorylation of STAT3 in Focal Cerebral Ischemia-Reperfusion Rat Model and its Role in Neuronal Apoptosis
}

\author{
Ke Guo ${ }^{1}$, Gang Yin ${ }^{2 *}$, Xiong-Hong $\mathrm{Zi}^{1}$ and Wen-Guang Yan ${ }^{3}$ \\ ${ }^{1}$ Department of Neurology, The Third Xiangya Hospital 410013, ${ }^{2}$ Department of Pathology, Xiangya Medical School, 410078, \\ ${ }^{3}$ Department of Rehabilitation, The Third Xiangya Hospital of Central South University, Changsha 410013, PR China \\ ${ }^{*}$ For correspondence: Email: docgk79@sina.com
}

Received: 16 April 2015

Revised accepted: 28 December 2015

\begin{abstract}
Purpose: To investigate the expression and tyrosine 705 phosphorylation of STAT3 in focal cerebral ischemia-reperfusion rat model and its role in neuronal apoptosis.

Methods: Ischemia-reperfusion model was established by thread-occluded method. Tetrazolium red (TTC), H/E and Nissl staining were used to evaluate whether ischemia-reperfusion model was successfully established. TUNEL staining and immunohistochemistry were employed to monitor apoptosis-positive nerve cells as well as STAT3-, p-Tyr705-STAT3-, Bcl-2- and Fas-positive cells in ischemic penumbra (IP) and ischemic core (IC).

Results: The results of TTC, HE and Nissl staining indicated that the ischemia-reperfusion model was successfully established. After $3 \mathrm{~h}$, ischemia followed by different reperfusion times, the STAT3-, $p$ Tyr705-STAT3-, Fas- and Bcl-2-positive cells counts and the apoptosis-positive nerve cells count were significantly $(p<0.05$ or 0.01$)$ increased to $27.20,29.20,15.90,18.50$, and 202.00 in IP and 19.50, $21.20,12.50$, 12.40, and 97.80 in IC, compared with the sham-operated group. As reperfusion times increased, cell counts did not decrease significantly relative to control group. Correlation analysis indicate that there was significant $(p<0.01)$ positive correlations among STAT3-, $p$-Tyr705-STAT3-, Fas- and Bcl-2-positive cells counts on the one hand, and apoptosis-positive nerve cells count in IP and IC, on the other hand.

Conclusion: Regulating expression and tyrosine 705 phosphorylation of STAT3 may be a new and effective strategy for treating cerebral infarction.
\end{abstract}

Keywords: Ischemia-reperfusion, Neuronal apoptosis, STAT3, Phosphorylation, Cerebral infarction

Tropical Journal of Pharmaceutical Research is indexed by Science Citation Index (SciSearch), Scopus, International Pharmaceutical Abstract, Chemical Abstracts, Embase, Index Copernicus, EBSCO, African Index Medicus, JournalSeek, Journal Citation Reports/Science Edition, Directory of Open Access Journals (DOAJ), African Journal Online, Bioline International, Open-J-Gate and Pharmacy Abstracts

\section{INTRODUCTION}

Cytokines and inflammatory mediators such as interleukin-6 (IL-6), interleukin-11 (IL-11), leukemia inhibitory factor (LIF) and ciliary neurotrophic factor (CNTF) are a few key factors in ischemic cerebrovascular disease [1-3]. Janus kinase-signal transducers and activators of transcription (JAK-STAT) signaling pathway plays an important role in regulating IL-6, IL-11,
LIF, CNTF, proliferation, differentiation, apoptosis and immunoregulation [4-9]. It is reported that STAT3, a STAT family member, can mediate apoptosis by regulating the expression levels of Bcl-2 and Bcl-xl, but Fas-mediated cell apoptosis signal inhibits the expression and phosphorylation of STAT3 $[10,11]$. It is reported that the expressions of STAT3 were increased after focal cerebral ischemia [12], but the mechanism is still unknown. Additionally, 
although the effect of focal cerebral ischemia on the STAT3 phosphorylation in ischemic penumbra (IP) and ischemic core (IC) has been reported [13], the effect of STAT3 phosphorylation on cell apoptosis and the correlations among STAT3 phosphorylation, cell apoptosis and apoptotic proteins (Bcl-2 and Fas) in focal cerebral ischemia are still unknown.

In this study, the effects of the STAT3 and its phosphorylation on neuronal apoptosis in focal cerebral ischemia-reperfusion rat model were investigated by detecting the expression levels of STAT3, STAT3 tyrosine 705 phosphorylation (pTyr705-STAT3), Fas, Bcl-2 and the apoptosispositive nerve cells count and analyzing the correlations among them.

\section{EXPERIMENTAL}

\section{Chemicals and reagents}

Rabbit anti STAT3 and p-Tyr705-STAT3 polyclonal antibodies were obtained from Santa Cruz (Santa Cruz, USA). Rabbit anti Bcl-2 and Fas antibodies, Ready-to-use strept avidin-biotin complex (SABC) immunohistochemical kit, phosphate buffer solution (PBS) and polylysine were purchased from BOSTER (Wuhan, China). Diaminobenzidine (DAB) chromogenic reagent kit and TUNEL staining kit were obtained from Zhongshan Biotechnology (Beijing, China). Tetrazolium red (TTC) was purchased from Sigma (St. Louis, MO, USA).

\section{Animals}

Healthy, adult and male Wistar rats $(300 \pm 20 \mathrm{~g})$ were provided by Laboratory Animal Centre, Xiangya School of Medicine, Central South University, Changsha, China. All procedures involving animals were conducted in accordance with the National Institute of Health Guide for the Care and Use of Laboratory Animals [14]. All experiments were performed with the approval of Ethics animals of Central South University in this study (protocol no. TXHCSU10491). Animals were housed in a room with the temperature of $25 \pm 2 \stackrel{\circ}{\circ}$, relative humidity of $50-60 \%$ and $12 \mathrm{~h}$ light/dark cycle, and had free access to food and water.

\section{Establishment of focal cerebral ischemia- reperfusion rat model}

Focal right middle cerebral artery ischemiareperfusion rat model was established by threadoccluded method $[15,16]$. Rats were firstly anaesthetized by $10 \%$ chloral hydrate $(0.35$
$\mathrm{mL} / 100 \mathrm{~g}$ ) intraperitoneally at $37 \pm 0.5 \stackrel{\circ}{\circ} \mathrm{C}$ and then fixed on surgery board in the supine position. A midline incision on the neck was made to expose the external carotid artery (ECA), right common carotid artery (RCCA) and internal carotid artery (ICA). Then the ECA and RCCA were ligated. The blood flow of ICA was blocked by pulling the preset string, and a Vnotch between bifurcation and the ligation of RCCA was cut. The nylon thread (length: $5.5 \mathrm{~cm}$; diameter: $0.25-0.28 \mathrm{~mm}$ ) was carefully introduced into the ICA lumen through the $\mathrm{V}$ notch until $2.0-2.3 \mathrm{~cm}$. The ICA was ligated to prevent the bleeding for $3 \mathrm{~h}$. Then bleeding was re-perfused for appropriate time by withdrawing the nylon thread. Sham-operated group were performed with the same surgical exposure procedures, but the carotid arteries were not blocked.

\section{Animal grouping and treatment}

45 Wistar rats were randomly divided into 9 groups $(n=5)$ : normal group (NG), shamoperated group (SG) and seven focal cerebral ischemia-reperfusion model groups ( $M G+0.5 \mathrm{~h}$, $3 \mathrm{~h}, 6 \mathrm{~h}, 12 \mathrm{~h}, 24 \mathrm{~h}, 3$ days or 7 days reperfusion). After observing, rats were anaesthetized by $10 \%$ chloral hydrate $(0.35$ $\mathrm{mL} / 100 \mathrm{~g}$ ) intraperitoneally and sacrificed.

\section{Neurological defect scoring}

After operation, the neurological statuses of rats were scored on a five-point scale [17]. Rats with no neurologic impairment scored 0 . Left forelimb of rats with adduction, flexion and failure to fully stretch scored 1. Rats with circling and rotating to left when crawling scored 2. Rats with toppling to the contralateral side when standing scored 3 . Rats with failure to walk spontaneously scored 4 . TTC, H/E, Nissl and TUNEL staining

After rats were sacrificed, their brains were harvested and frozen at $-20^{\circ} \mathrm{C}$. After $20 \mathrm{~min}$, the coronal plane was obtained from brain via a tissue slicer (AO, USA) and stained with $2 \%$ TTC staining solution at $3{ }^{\circ} \mathrm{O}$ for $30 \mathrm{~min}$. The remaining brain tissue samples were fixed in $4 \%$ paraformaldehyde for $6 \mathrm{~h}$. Then the IP of the fixed brain tissue was treated with the following steps: dehydration, transparency, dip in wax, embedding and slicing. The paraffin sections were stained by Harris hematoxylin and eosin $(\mathrm{H} / \mathrm{E})$ staining solution, $1 \mathrm{~g} / \mathrm{L}$ Nissl staining solution and TUNEL staining kit, respectively. Subsequently, the H/E staining, Nissl staining and TUNEL staining slicing were observed and photographed by microscope. The apoptosispositive nerve cells count in TUNEL staining 
slicing was analyzed by HPIAS-100 highdefinition color pathological image analysis system (Wuhan, China).

\section{Immunohistochemical staining for STAT3, p- Tyr705-STAT3, Bcl-2 and Fas}

The coronal plane was obtained from the fixed brain tissue, and then focal side and uninjured side were picked out from coronal plane. Further, IC and IP were picked out from focal side, and meanwhile, the counterpart of the ischemic brain (CIB) was obtained. According to method as above described, the different tissues were made into paraffin section. After heat-mediated antigen retrieval, paraffin sections were incubated with STAT3, p-Tyr705-STAT3, Bcl-2 and Fas antibodies and subsequently with $S A B C$ immunohistochemical kit. After staining by $D A B$ chromogenic reagent kit, the paraffin sections were observed and analyzed by microscope and HPIAS-100 high-definition color pathological image analysis system (Wuhan, China).

\section{Statistical analysis}

All data are presented as mean \pm standard deviation. Differences among different groups were analyzed by one-way ANOVA (LSD or Games-Howell test) on SPSS 21.0. Correlations among the apoptosis-positive nerve cells count and the STAT3-, p-Tyr705-STAT3-, Bcl-2- and Fas-positive cells counts in IP and IC were analyzed by two-tails Pearson or Spearman test on SPSS 21.0. When $p$-value was lower than 0.05 or 0.01 , differences were considered statistically significant.

\section{RESULTS}

\section{Neuro-behavioral observation}

Neurological defect scoring was used to evaluate whether the focal cerebral ischemia modeling was successfully established. When the neurological defect scoring was 1,2 or 3 , rat was selected out as experimental animals. When the neurological defect scoring was 0 or 4 , it indicated that the focal cerebral ischemia modeling was unsuccessfully established. NG and $S G$ did not have neurological impairment. The unqualified focal cerebral ischemia rats were replaced with standby animals.

\section{TTC staining}

After staining with TTC, the color of ischemic tissue was pale, and the color of normal tissue was red. As shown in Figure 1, the color of the coronal plane in the $\mathrm{NG}$ and $\mathrm{SG}$ was red, and the color of the coronal plane in the MG was pale.

\section{H/E and Nissl staining}

As shown in Figure 2, the form and construction of brain nerve cells, spongiocyte and capillaries in $N G$ and $S G$ were normal and intact, and there was no red dye in the cytoplasm. After $3 \mathrm{~h}$ ischemia followed by 0.5 or $3 \mathrm{~h}$ reperfusion, there was not evident changes in the form and construction of brain nerve cells, spongiocyte and capillaries.

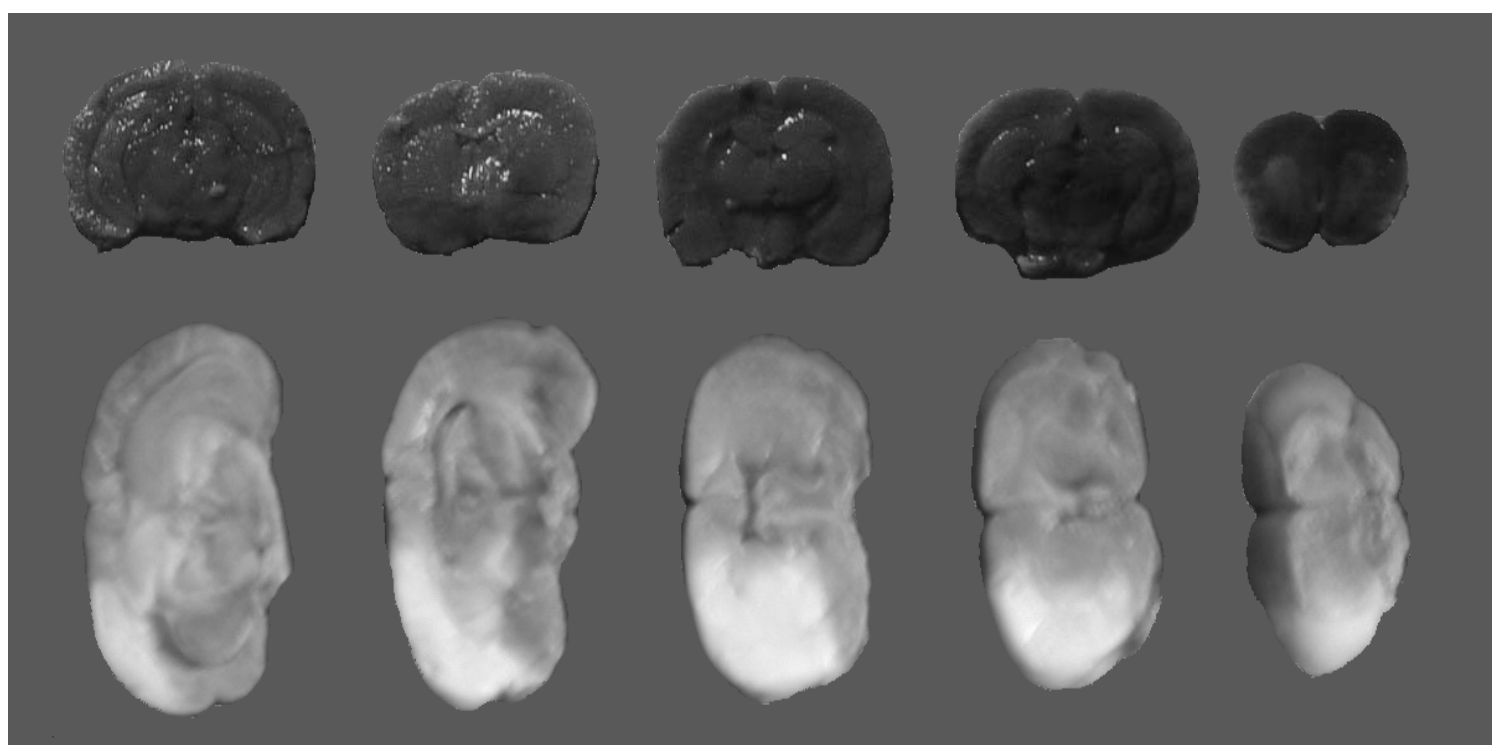

Figure 1: TTC staining of coronal plane in the NG, SG and MG; the red coronal plane in the NG and SG; the pale coronal plane in the MG 
With the increase of reperfusion time, necrocytosis and cell losses were evidently observed. When the reperfusion time was $12 \mathrm{~h}$, the mild interstitial edema was observed. When the reperfusion time was $24 \mathrm{~h}$, the moderate interstitial edema, the reduction of cell count, the swelling and irregular cellular morphology and the inflammatory cell infiltration were evidently observed in $\mathrm{HE}$ staining. Furthermore, the karyopyknosis, the shrink and deformation of soma, the losses of Nissl's body and ghost cell were evidently observed in Nissl staining. When the reperfusion time was 3 or 7 days, extensive neuronal deaths near the pyramidal cells and corpus striatum of cerebral cortex were evidently observed.

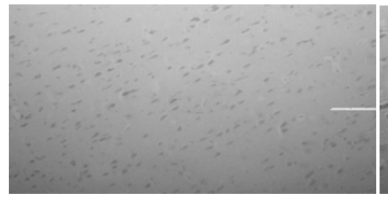

HE staining, NG or SG $\times 100$

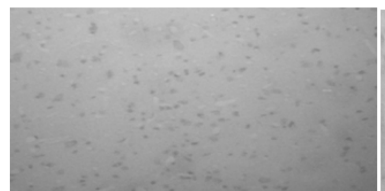

HE staining, MG $\times 100$ reperfusion $24 \mathrm{~h}$

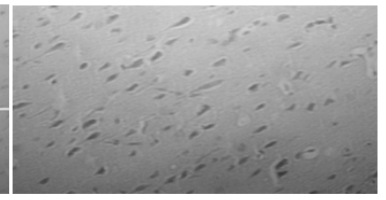

HE staining, MG $\times 100$ reperfusion $12 \mathrm{~h}$

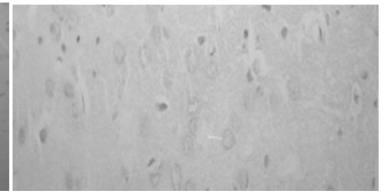

HE staining, MG $\times 100$ reperfusion $3 \mathrm{~d}$

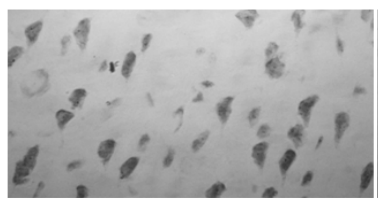

Nissl staining, NG or SG $\times 400$

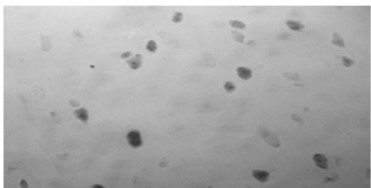

Nissl staining, MG $\times 400$ reperfusion $24 \mathrm{~h}$

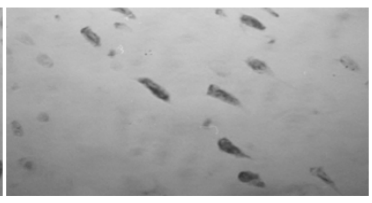

Nissl staining, MG $\times 400$ reperfusion $12 \mathrm{~h}$

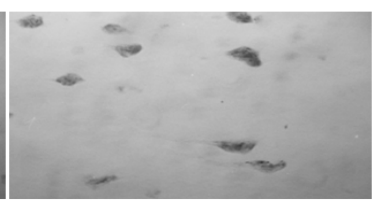

Nissl staining, MG $\times 400$ reperfusion $3 \mathrm{~d}$

Figure 2: $\mathrm{HE}$ and Nissl staining of IP in the NG, SG and MG

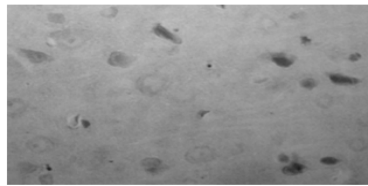

TUNEL staining, NG or SG $\times 400$

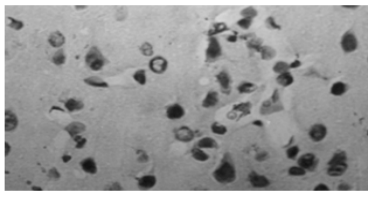

TUNEL staining, MG $\times 400$ reperfusion $12 \mathrm{~h}$

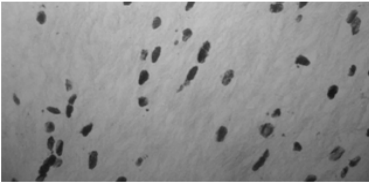

TUNEL staining, MG $\times 400$ reperfusion $0.5 \mathrm{~h}$

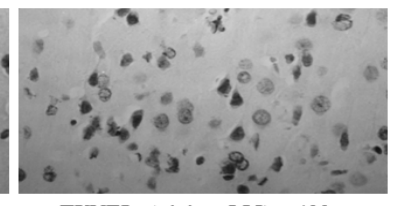

TUNEL staining, MG $\times 400$ reperfusion $24 \mathrm{~h}$

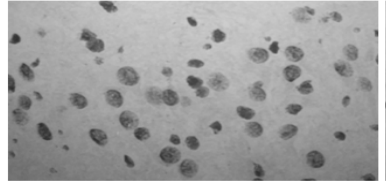

TUNEL staining, MG $\times 400$ reperfusion $3 \mathrm{~h}$

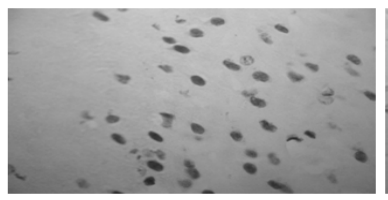

TUNEL staining, MG $\times 400$ reperfusion $3 \mathrm{~d}$

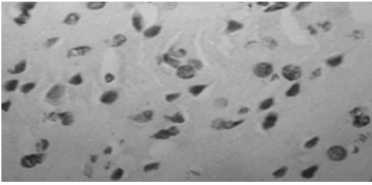

TUNEL staining, MG $\times 400$ reperfusion $6 \mathrm{~h}$

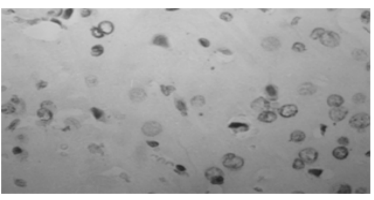

TUNEL staining, MG $\times 400$ reperfusion $7 \mathrm{~d}$

Figure 3: TUNEL staining of ischemic penumbra

Table 1: Effect of focal cerebral ischemia-reperfusion on apoptosis-positive nerve cells count in IP, IC and CIB

\begin{tabular}{|c|c|c|c|}
\hline Group & IP & IC & CIB \\
\hline NG & $4.50 \pm 0.97$ & $4.10 \pm 1.20$ & $3.80 \pm 0.79$ \\
\hline SG & $6.20 \pm 1.32$ & $6.40 \pm 1.71$ & $3.60 \pm 0.70$ \\
\hline$M G+0.5 \mathrm{~h}$ reperfusion & $120.90 \pm 11.09^{* \#}$ & $65.40 \pm 4.06^{\approx \#}$ & $3.10 \pm 0.74$ \\
\hline$M G+3$ h reperfusion & $202.00 \pm 9.92$ & $97.80 \pm 3.68$ & $3.70 \pm 1.16$ \\
\hline$M G+6$ h reperfusion & $194.10 \pm 6.03$ & $93.60 \pm 3.80$ & $3.40 \pm 0.97$ \\
\hline$M G+12 h$ reperfusion & $129.80 \pm 3.74^{\text {\# }}$ & $75.80 \pm 5.69^{\prime \#}$ & $4.20 \pm 1.55$ \\
\hline$M G+24 h$ reperfusion & $102.70 \pm 5.62^{x \#}$ & $63.90 \pm 4.84^{\text {\# }}$ & $4.00 \pm 1.05$ \\
\hline MG + 3 days reperfusion & $74.50 \pm 6.90^{\pi \#}$ & $35.60 \pm 5.68^{\star \#}$ & $3.90 \pm 0.88$ \\
\hline MG +7 days reperfusion & $6.50 \pm 1.08^{\#}$ & $6.00 \pm 1.05^{\#}$ & $3.50 \pm 1.51$ \\
\hline
\end{tabular}

Trop J Pharm Res, February 2016; 15(2): 270 
Effect of focal cerebral ischemia-reperfusion on STAT3-, p-Tyr705-STAT3-, Bcl-2- and Faspositive cells counts

As shown in Tables 2 - 5, the STAT3-, p-Tyr705STAT3-, Fas- and Bcl-2-positive cells counts were not significantly different between the NG and SG, and it indicated that the STAT3-, pTyr705-STAT3-, Fas- and Bcl-2-positive cells counts were not affected by the sham-operation. After $3 \mathrm{~h}$ ischemia followed by different reperfusion times, the STAT3-, p-Tyr705-STAT3, Fas- and Bcl-2-positive cells counts in IP and IC were significantly $(p<0.05$ or 0.01$)$ increased and then decreased to no significant difference, compared with the SG. Additionally, there were a few STAT3-, p-Tyr705-STAT3- and Bcl-2-positive cells were detected in CIB.

Table 2: Effect of focal cerebral ischemia-reperfusion on STAT3-positive cells count in IP, IC and CIB

\begin{tabular}{|c|c|c|c|}
\hline Group & IP & IC & CIB \\
\hline $\mathrm{NG}$ & $0.00 \pm 0.00$ & $0.00 \pm 0.00$ & $0.00 \pm 0.00$ \\
\hline SG & $0.00 \pm 0.00$ & $0.00 \pm 0.00$ & $0.00 \pm 0.00$ \\
\hline$M G+0.5 \mathrm{~h}$ reperfusion & $3.60 \pm 0.97^{* \#}$ & $3.40 \pm 0.97^{\star \#}$ & $2.60 \pm 0.97^{* \#}$ \\
\hline$M G+3 h$ reperfusion & $5.40 \pm 0.84^{* \#}$ & $3.50 \pm 0.85^{\star \#}$ & $2.10 \pm 0.99^{* \#}$ \\
\hline$M G+6 h$ reperfusion & $6.70 \pm 1.16^{* \#}$ & $4.60 \pm 1.07^{* \#}$ & $4.40 \pm 1.07^{* \#}$ \\
\hline$M G+12 \mathrm{~h}$ reperfusion & $10.20 \pm 1.48^{* \#}$ & $8.20 \pm 1.32^{* \#}$ & $5.40 \pm 1.07^{* \#}$ \\
\hline$M G+24$ h reperfusion & $27.20 \pm 1.55^{\star}$ & $19.50 \pm 1.58^{\star}$ & $7.80 \pm 1.48^{*}$ \\
\hline MG + 3 days reperfusion & $20.90 \pm 2.33^{*} \#$ & $14.70 \pm 2.67^{* \#}$ & $7.40 \pm 2.95^{\star}$ \\
\hline MG +7 days reperfusion & $14.30 \pm 2.16^{* \#}$ & $14.10 \pm 1.59^{* \#}$ & $4.70 \pm 0.95^{* \#}$ \\
\hline
\end{tabular}

Table 3: Effect of focal cerebral ischemia-reperfusion on p-Tyr705-STAT3-positive cells count in IP, IC and CIB

\begin{tabular}{lccc}
\hline Group & IP & IC & CIB \\
\hline NG & $0.00 \pm 0.00$ & $0.00 \pm 0.00$ & $0.00 \pm 0.00$ \\
$S G$ & $0.00 \pm 0.00$ & $0.00 \pm 0.00$ & $0.00 \pm 0.00$ \\
$M G+0.5$ h reperfusion & $3.20 \pm 1.03^{* \#}$ & $3.10 \pm 0.74^{* \#}$ & $2.40 \pm 1.07^{*}$ \\
$M G+3$ h reperfusion & $5.40 \pm 1.58^{* \#}$ & $21.20 \pm 3.01^{*}$ & $2.20 \pm 1.32^{*}$ \\
$M G+6$ h reperfusion & $11.00 \pm 2.16^{* \#}$ & $19.70 \pm 2.98^{*}$ & $3.90 \pm 0.99^{*}$ \\
$M G+12$ h reperfusion & $20.90 \pm 3.00^{* \#}$ & $11.40 \pm 2.59^{* \#}$ & $5.80 \pm 1.23^{*}$ \\
$M G+24$ h reperfusion & $29.20 \pm 1.69^{*}$ & $8.40 \pm 1.51^{* \#}$ & $9.90 \pm 1.79^{*}$ \\
$M G+3$ days reperfusion & $15.90 \pm 1.37^{* \#}$ & $5.90 \pm 0.88^{* \#}$ & $6.30 \pm 0.95^{*}$ \\
$M G+7$ days reperfusion & $5.10 \pm 1.20^{*}$ & $3.20 \pm 1.32^{* \#}$ & $3.00 \pm 1.41^{*}$
\end{tabular}

${ }^{*} P<0.01$, compared with the SG; ${ }^{\#} p<0.01$, compared with the $M G+24 h$ reperfusion in IP and the $M G+3 h$ reperfusion in IC

Table 4: Effect of focal cerebral ischemia-reperfusion on Bcl-2-positive cells count in IP, IC and CIB

\begin{tabular}{|c|c|c|c|}
\hline Group & IP & IC & CIB \\
\hline$N G$ & $4.70 \pm 1.16$ & $4.20 \pm 1.03$ & $4.20 \pm 1.23$ \\
\hline SG & $4.90 \pm 0.88$ & $4.10 \pm 0.99$ & $3.90 \pm 0.87$ \\
\hline$M G+0.5 \mathrm{~h}$ reperfusion & $5.00 \pm 0.67^{\#}$ & $5.20 \pm 0.79^{\#}$ & $4.10 \pm 0.74$ \\
\hline$M G+3 h$ reperfusion & $6.40 \pm 0.97^{\mathbf{\Delta} \#}$ & $5.60 \pm 1.17^{* \#}$ & $4.30 \pm 0.82$ \\
\hline$M G+6 h$ reperfusion & $13.70 \pm 1.49^{*}$ & $12.50 \pm 1.35^{*}$ & $4.30 \pm 0.95$ \\
\hline$M G+12 \mathrm{~h}$ reperfusion & $15.90 \pm 1.67^{*}$ & $10.60 \pm 1.65^{* \#}$ & $4.50 \pm 0.85$ \\
\hline$M G+24$ h reperfusion & $11.20 \pm 1.69^{*} \#$ & $9.00 \pm 1.33^{* \#}$ & $4.90 \pm 0.74^{\wedge}$ \\
\hline MG + 3 days reperfusion & $10.10 \pm 1.91^{*}$ & $7.10 \pm 1.20^{*}$ & $3.50 \pm 1.08$ \\
\hline MG +7 days reperfusion & $4.60 \pm 0.84^{\#}$ & $4.70 \pm 0.82^{\#}$ & $4.60 \pm 1.07$ \\
\hline
\end{tabular}

$\triangle P<0.05, * 0<0.01$, compare
$M G+6$ h reperfusion in IC 
Table 5: Effect of focal cerebral ischemia-reperfusion on Fas-2-positive cells count in IP, IC and CIB

\begin{tabular}{|c|c|c|c|}
\hline Group & IP & IC & CIB \\
\hline $\mathrm{NG}$ & $0.00 \pm 0.00$ & $0.00 \pm 0.00$ & $0.00 \pm 0.00$ \\
\hline SG & $0.00 \pm 0.00$ & $0.00 \pm 0.00$ & $0.00 \pm 0.00$ \\
\hline$M G+0.5 \mathrm{~h}$ reperfusion & $3.20 \pm 1.32^{\approx}$ & $2.80 \pm 0.92^{\pi \#}$ & $0.00 \pm 0.00$ \\
\hline$M G+3 h$ reperfusion & $3.70 \pm 0.95^{\#}$ & $3.90 \pm 1.20^{\#}$ & $0.00 \pm 0.00$ \\
\hline$M G+6 h$ reperfusion & $8.20 \pm 1.35^{\#}$ & $7.70 \pm 1.49^{\#}$ & $0.00 \pm 0.00$ \\
\hline$M G+12 \mathrm{~h}$ reperfusion & $13.20 \pm 1.75^{* \#}$ & $12.40 \pm 1.65$ & $0.00 \pm 0.00$ \\
\hline $\begin{array}{l}M G+24 \text { h reperfusion } \\
M G+3 \text { days reperfusion } \\
M G+7 \text { days reperfusion }\end{array}$ & $\begin{array}{l}18.50 \pm 1.96^{\star} \\
9.40 \pm 1.51^{\#} \\
1.80 \pm 0.92^{\#}\end{array}$ & $\begin{array}{l}8.20 \pm 1.32^{\star \#} \\
7.60 \pm 1.51^{\star \#} \\
1.20 \pm 0.79^{\star \#}\end{array}$ & $\begin{array}{l}0.00 \pm 0.00 \\
0.00 \pm 0.00 \\
0.00 \pm 0.00\end{array}$ \\
\hline
\end{tabular}

${ }^{*} P<0.01$, compared with the SG; ${ }^{\#} p<0.01$, compared with the $M G+24 h$ reperfusion in IP and the $M G+12 h$ reperfusion in IC

Table 6: Correlations among the STAT3-, p-Tyr705-STAT3-, Bcl-2-, Fas-positive cells and the apoptosis-positive nerve cells counts (APNCC) in IP and IC

\begin{tabular}{|c|c|c|c|c|c|c|c|c|c|c|}
\hline \multirow{2}{*}{$\begin{array}{l}\text { Variable } \\
\text { Part }\end{array}$} & \multicolumn{2}{|c|}{ STAT3 } & \multicolumn{2}{|c|}{ p-Tyr705-STAT3 } & \multicolumn{2}{|c|}{ Bcl-2 } & \multicolumn{2}{|c|}{ Fas } & \multicolumn{2}{|c|}{ APNCC } \\
\hline & IP & IC & IP & IC & IP & IC & IP & IC & IP & IC \\
\hline STAT3 & & & & & & & & & 0.179 & 0.159 \\
\hline$P$ & & & & & & & & & 0.091 & 0.135 \\
\hline p-Tyr705-STAT3 & 0.445 & 0.385 & & & & & & & 0.445 & 0.886 \\
\hline$P$ & 0.000 & 0.000 & & & & & & & 0.000 & 0.000 \\
\hline $\mathrm{Bcl}-2$ & 0.524 & 0.488 & 0.776 & 0.693 & & & & & 0.576 & 0.606 \\
\hline$P$ & 0.000 & 0.000 & 0.000 & 0.000 & & & & & 0.000 & 0.000 \\
\hline Fas & 0.685 & 0.528 & 0.907 & 0.707 & 0.825 & 0.822 & & & 0.549 & 0.658 \\
\hline$P$ & 0.000 & 0.000 & 0.000 & 0.000 & 0.000 & 0.000 & & & 0.000 & 0.000 \\
\hline
\end{tabular}

Correlations among the STAT3-, p-Tyr705STAT3-, Bcl-2- and Fas-positive cells counts and the apoptosis-positive nerve cells count in IP and IC

The correlations among STAT3-, p-Tyr705STAT3-, Bcl-2- and Fas-positive cells counts and the apoptosis-positive nerve cells count in seven focal cerebral ischemia-reperfusion model groups (MG +0.5 h, 3 h, 6 h, 12 h, 24 h, 3 days or 7 days reperfusion) were analyzed by two-tails Pearson or Spearman test. As shown in Table 6, there are significantly $(p<0.01)$ positive correlations among STAT3-, p-Tyr705-STAT3-, Fas- and Bcl-2-positive cells counts in IP and IC, and the p-Tyr705-STAT3-, Fas- and Bcl-2positive cells counts in IP and IC were significantly $(p<0.01)$ and positively related to the apoptosis-positive nerve cells count.

\section{DISCUSSION}

The focal cerebral artery ischemia-reperfusion rat model is a commonly used model to investigate ischemia-reperfusion-related problems [18]. TTC, $\mathrm{HE}$ and Nissl staining are three commonly used methods to explore whether the focal middle cerebral artery ischemia-reperfusion rat model is successfully established $[19,20]$. The results of TTC, HE and Nissl staining indicated that the focal middle cerebral artery ischemia-reperfusion rat model was successfully established.

Ischemia brain tissue can be divided into IP and IC, whose sizes are in dynamic change [21]. Generally, necrosis is the main pathway of nerve cell in IC, but apoptosis is the main pathway of nerve cell in IP [22,23]. The necrosis and apoptosis in cerebral ischemia are related to the duration or degree of ischemia [17,24]. In the present study, TUNEL staining was used to study the effect of ischemia-reperfusion on the apoptosis-positive nerve cells count in IP and IC. The results of TUNEL staining indicated that after focal cerebral artery ischemia, the apoptosispositive nerve cells count was increased in IP and IC, and the increase was reversed by reperfusion.

The inhibition of JAK-STAT signaling induces the apoptosis of cancer cell apoptosis via death receptor- and mitochondria-mediated apoptotic pathways $[25,26]$. Fas, a pro-apoptotic protein, plays an important role in death receptormediated apoptotic pathway [27]. Bcl-2, an antiapoptotic protein, plays an important role in mitochondria-mediated apoptotic pathway [28]. The increase of the expressions of STAT3 and pTyr705-STAT3 proteins can induce the JAKSTAT signaling. In the present study, after $3 \mathrm{~h}$ ischemia followed by different reperfusion times, the expressions of STAT3, p-Tyr705-STAT3, Fas 
and $\mathrm{Bcl}-2$ proteins were first increased and then decreased in IP and IC. The correlation analysis indicated that there are positive correlations among STAT3, p-Tyr705-STAT3, Fas and Bcl-2positive cells counts, and the p-Tyr705-STAT3, Fas and Bcl-2-positive cells counts were positively related to the apoptosis-positive nerve cells count. These results indicated that the reversed mechanisms of reperfusion on the ischemia-induced nerve cells apoptosis were related to the interactions among STAT3, pTyr705-STAT3, Fas and Bcl-2 proteins.

\section{CONCLUSION}

The findings of this study show that regulating expression and tyrosine 705 phosphorylation of STAT3 may be a new effective strategy for treating cerebral infarction. Furthermore, the findings to further investigate the molecular pathological mechanism of focal cerebral ischemia.

\section{CONFLICT OF INTEREST}

The authors declare that there is no conflict of interest associated with this work.

\section{CONTRIBUTION OF AUTHORS}

We declare that this work was done by the authors named in this article and all liabilities pertaining to claims relating to the content of this article will be borne by the authors. Ke Guo and Gang Yin conceived and designed the study. Ke Guo, Xiong-Hong $\mathrm{Zi}$ and Wen-Guang Yan collected and analyzed the data.

\section{REFERENCES}

1. Rodríguez-Yáñez M, Castillo J. Role of inflammatory markers in brain ischemia. Curr Opin Neurol 2008; 21(3): 353-357.

2. Yoshikawa A, Kamide T, Hashida K, Ta HM, Inahata $Y$, Takarada-lemata M, Hattori $T$, Mori K, Takahashi $R$, Matsuyama $T$, et al. Deletion of Atf6a impairs astroglial activation and enhances neuronal death following brain ischemia in mice. J Neurochem 2015; 132(3): 342-353.

3. Mengozzi M, Ermilov P, Annenkov A, Ghezzi P, Pearl F. Definition of a family of Tissue-protective cytokines using functional cluster analysis: a proof-of-concept study. Front Immunol 2014; 5: 115.

4. Harrison DA. The JAK/STAT pathway. Cold Spring Harb Perspect Biol 2012; 4: a011205.

5. Kisseleva T, Bhattacharya S, Braunstein J, Schindler $C W$. Signaling through the JAK/STAT pathway, recent advances and challenges. Gene 2002; 285: 1-24.
6. Shuai K. Serine Phosphorylation: arming Statl against Infection. Immunity 2003; 19: 771-772.

7. Ramana CV, Gil MP, Schreiber RD, Stark GR. Statdependent and -independent pathways in IFN-gammadependent signaling. Trends Immunol 2002; 23: 96-101.

8. Bolli $R$, Dawn B, Xuan YT. Role of the JAK-STAT pathway in protection against myocardial ischemia/reperfusion injury. Trends Cardiovasc Med 2003; 13: 72-79.

9. Villarino $A V$, Kanno $Y$, Ferdinand JR, O'Shea JJ. Mechanisms of Jak/STAT signaling in immunity and disease. J Immunol 2015; 194(1): 21-27.

10. Zaanan A, Okamoto K, Kawakami H, Khazaie K, Huang $S$, Sinicrope FA. The mutant KRAS gene up-regulates $B c l-x I$ protein via STAT3 to confer apoptosis resistance that is reversed by Bim protein induction and Bcl-xl antagonism. J Biol Chem 2015; 290(39): 23838-23849.

11. Cucurbitacin $E$ induces $G(2) / M$ phase arrest through STAT3/p53/p21 signaling and apoptosis via Fas/CD95 and mitochondria-dependent pathways in human bladder cancer T24 cells. Evid Based Complement Alternat Med 2012; 2012: 952762.

12. Chen Z, Li L, Mo X, Zhang L, Xie Y, Guo Q, Wang Y. Non-invasive remote limb ischemic postconditioning protects rats against focal cerebral ischemia by upregulating STAT3 and reducing apoptosis. Int $\mathrm{J} \mathrm{Mol} \mathrm{Med}$ 2014; 34(4): 957-966.

13. Suzuki S, Tanaka K, Nogawa S, Dembo T, Kosakai A, Fukuuchi Y. Phosphorylation of Signal Transducer and Activator of Transcription-3 (Stat3) after Focal Cerebral Ischemia in Rats. Exp Neurol 2001: 170: 63-71.

14. The National Research Council of The National Academy of Sciences. Guide for the Care and Use of Laboratory Animals: Eight Edition. Washington, D.C.: The National Academies Press; 2010.

15. Chitnis $T$, Najafian N, Benou C, Salama AD, Grusby MJ, Sayegh MH, Khoury SJ. Effect of targeted disruption of STAT4 and STAT6 on the induction of experimental autoimmune encephalomyelitis. J Clin Invest 2001; 108: 739-747.

16. Lentsch AB, Kato A, Davis B, Wang W, Chao C, Edwards MJ. STAT4 and STAT6 regulate systemic inflammation and protect against lethal endotoxemia. J Clin Invest 2001; 108: 1475-1482.

17. Longa EZ, Weinstein PR, Carlson S, Cummins $R$. Reversible middle cerebral artery occlusion without craniectomy in rats. Stroke 1989; 20: 84-91.

18. Zhu J, Guo L, Watson CJ, Hu-Li J, Paul WE. Stat6 is necessary and sufficient for IL-4's role in Th2 differentiation and cell expansion. J Immunol 2001; 166: 7276-7281.

19. Kramer M, Dang J, Baertling F, Denecke B, Clarner T, Kirsch $C$, Beyer C, Kipp M. TTC staining of damaged brain areas after MCA occlusion in the rat does not constrict quantitative gene and protein analyses. J Neurosci Methods 2010; 187: 84-89.

20. Liu C, Shi Z, Fan L, Zhang C, Wang $K$, Wang $B$. Resveratrol improves neuron protection and functional

Trop J Pharm Res, February 2016; 15(2): 273 
recovery in rat model of spinal cord injury. Brain Res 2011; 1374: 100-109.

21. De-Fraja C, Conti L, Govoni S, Battaini F, Cattaneo E. STAT signalling in the mature and aging brain. Int $J$ Dev Neurosci 2000; 18: 439-446.

22. Takagi Y, Harada J, Chiarugi A, Moskowitz MA. STAT1 is activated in neurons after ischemia and contributes to ischemic brain injury. J Cereb Blood Flow Metab 2002; 22:1311-1318.

23. Stephanou A, Brar BK, Knight RA, Latchman DS. Opposing actions of STAT-1 and STAT-3 on the Bel-2 and Bcl-x promoters. Cell Death Differ 2000; 7: 329-330.

24. Suzuki S, Tanaka K, Nogawa S, Dembo T, Kosakai A, Fukuuchi Y. Phosphorylation of Signal Transducer and Activator of Transcription-3 (Stat3) after Focal Cerebral Ischemia in Rats. Exp Neurol 2001; 170: 63-71.
25. Du W, Hong J, Wang YC, Zhang YJ, Wang $P$, Su YW, Lin YW, Lu R, Zou WP, Xiong $H$, et al. Inhibition of JAK2/STAT3 signalling induced colorectal cancer cell apoptosis via mitochondrial pathway. J Cell Mol Med 2012; 16(8): 1878-1888.

26. Jo M, Park MH, Kollipara PS, An BJ, Song HS, Han SB, Kim JH, Song MJ, Hong JT. Anti-cancer effect of bee venom toxin and melittin in ovarian cancer cells through induction of death receptors and inhibition of JAK2/STAT3 pathway. Toxicol Appl Pharm 2012; 258(1): 72-81.

27. Ouyang L, Shi Z, Zhao S, Wang FT, Zhou TT, Liu B, Bao JK. Programmed cell death pathways in cancer: a review of apoptosis, autophagy and programmed necrosis. Cell Prolif 2012; 45(6): 487-498.

28. Shi Y. A structural view of mitochondria-mediated apoptosis. Nat Struct Biol 2001; 8(5): 394-401. 